\section{Dental Materials Effect in Neutron Contamination: Electron Mode of a Linac}

\author{
Ghorbani M. ${ }^{1 \oplus}$, Ebrahimi Bardar M.**(i)
}

\begin{abstract}
Background: Neutron contamination is produced in electron beams of linac when tooth or dental materials are located in the path of beam.
\end{abstract}

Objective: This study aims to determine the neutron dose contamination from different dental restoration materials in electron mode of a linac.

Material and Methods: In this experimental study, the neutron dose contamination was calculated in the presence of tooth and tooth restored by Ceramco C3 veneer, Eclipse or amalgam. The electron mode included 8, 12, and $14 \mathrm{MeV}$ electron beams of Siemens Primus linac at different depths before and after tooth. MCNPX code was used to simulate the linear accelerator and dental restoration materials. Tooth and tooth restoration materials were located in the beams' central axis and the neutron dose was scored in $3 \times 3 \times 1 \mathrm{~cm}^{3}$ voxels at different depths before and after the tooth.

Results: The highest neutron dose contamination was observed for the combination of the tooth and Eclipse in 12 and $14 \mathrm{MeV}$ beams and the maximum calculated relative neutron dose was 1.53 for tooth and Eclipse for $14 \mathrm{MeV}$ electron beam.

Conclusion: Tooth and dental materials lead to neutron dose contamination production, therefore, in order to avoid having harmful effects on normal tissues due to the neutron beam in head and neck cancer, it is recommended that treatment planning performed should not place tooth with dental restoration materials in the path of the beam and lower energy electron beams be used.

Citation: Ghorbani M, Ebrahimi Bardar M. Dental Materials Effect in Neutron Contamination: Electron Mode of a Linac. J Biomed Phys Eng. 2020;10(2):155-160. doi: 10.31661/jbpe.v0i0.920.

\section{Keywords}

Siemens Primus linac; Electron Beam; Tooth; Restoration Material; Neutrons; Monte Carlo Method

\section{Introduction}

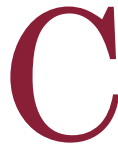

ancer is one of the most important causes of mortality [1]. Head and neck cancer are accounted as about $4 \%$ of all cancers [2]. In recent decades, modalities for the treatment of cancer have increased dramatically. Cancer treatment may involve one procedure such as surgery, chemotherapy, immunotherapy and radiation therapy or a combination of them [3]. Among these modalities, radiation therapy is well known as one of the most important methods used for the treatment for different types of cancers. Radiotherapy is based on the interaction of ionizing radiation with tumor cells and the resulted biological effects lead to controlling and providing the treatment of cancerous tissues.

Energy and intensity of radiation used in radiotherapy should be enough to cause harmful biological effects and destroy cancer cells. In addition to the beneficial effects of radiation, it also has undesirable effects on normal tissues in such a way that cells with higher proliferation rate show more sensitivity to ionizing radiation [4]. Radiation therapy,
${ }^{1} \mathrm{PhD}$, Biomedical Engineering and Medical

Physics Department,

Faculty of Medicine,

Shahid Beheshti Univer-

sity of Medical Sciences,

Tehran, Iran

${ }^{2} \mathrm{MSc}$, Department of

Medical Physics, Faculty

of Medicine, Mashhad

University of Medical Sci-

ences, Mashhad, Iran

*Corresponding author:

M. Ebrahimi Bardar

Medical Physics Depart-

ment, Faculty of Medi-

cine, Mashhad Univer-

sity of Medical Sciences,

Mashhad, Iran

E-mail: me_8471@

yahoo.com

Received: 16 March 2018 Accepted: 8 May 2018 
as a common treatment for head and neck cancers, is employed in both forms of internal and external beams, and in $90 \%$ of treatments beams are emitted from a source outside the body [5].

A linear accelerator (linac) is the most common tool used for external beam radiation treatment of cancer patients. Charged particles like electrons are accelerated in a linear tube and take energy. The accelerated electrons are used either directly for treatment of superficial tumors or indirectly after they hit a target, and then secondary produced X-rays can treat deep tumors. In other words, these accelerators can be used in both electron and photon modes [6].

In the 1970s, high energy linear accelerators with the ability to produce photons and electrons with different energies were increasingly available for clinical uses [7-8]. Increasing commercial development of linacs with clinical experience achieved in a number of leading centers for radiation therapy show that there is no substitution for electron beam therapy in some common cases, due to advantages such as uniformity of dose in the target volume and the dose reduction in deeper tissues.

In electron mode, there are two important interactions which produce neutron contamination. The first is electron capture, a phenomenon in which orbital electrons are captured by the nucleus and the protons are converted to neutron, and the latter is photonuclear interaction of the secondary photons generated by electrons that result in neutron emission from the nucleus [9]. Neutrons are produced by collisions of electrons and high-energy photons with different target materials, the flattening filter, collimators and other components which exist in the path of beam.

During electron-beam radiation therapy, patients with head and neck cancers, who have dental restoration materials, are at risk of exposure to neutron contamination. These heterogeneities are often presented in the environment of treatment volumes and can lead to neutron contamination. When high energy electrons hit these materials, secondary electrons are scattered and photon contamination is produced and then photonuclear interactions of the secondary photons may result in neutron contamination. Radiosensitive organs in the head and neck, including the salivary glands, eyes and spinal cord that they can be affected due to this process and as a result, this may have different side effects in the treatment area [10]. The results of a previous study showed that the change in the electron dose depends on the energy of electron beam and the type of dental restoration material [11]. For the tooth filled with amalgam and by 14 $\mathrm{MeV}$ electron beam, the electron dose had 7\% enhancement as a result of electron backscattering from tooth and amalgam. To prevent the backscattered electron emission from the tooth restored with a restoration material, which can enhance the dose received by the surrounding tissues, the use of bolus around the soft tissue is recommended. The interaction of the electron with the tooth and dental materials, due to dependence of electron dose on depth, kind of heterogeneity and beam energy causes changes in electron dose before and after the tooth. These changes in electron dose distribution, when the tooth is irradiated by the electron beam in cancer of head and neck, should be considered in the process of treatment planning of patients. There are studies evaluating the contribution of photon contamination in the radiation therapy by the electron beam due to the dental restoration [11], however the previous studies have not calculated the neutron contamination of electron beams of a linac in the presence of dental materials. This study aims to calculate the neutron contamination of electron beams with different amounts of energies in the case of various dental restoration materials.

\section{Material and Methods}

Monte Carlo simulations

In this experimental study, calculations were 
Dental Restorations-neutron Contamination

performed for 8 and 12 and $14 \mathrm{MeV}$ electron modes of a Siemens Primus linear accelerator, and $10 \times 10 \mathrm{~cm}^{2}$ applicator using MCNPX (version 2.6.0) simulation code.

The verification of the linac's simulation was based on previous investigations [12]. In that study, 8 and 12 and $14 \mathrm{MeV}$ electron modes were studied and the percentages of depth dose values were calculated for $10 \times 10,15 \times 15$ and $25 \times 25 \mathrm{~cm}^{2}$ applicators. The gamma index was used to compare the dose distribution obtained from simulation and measurement, for which the values for various energies and applicators were reported less than unity. This showed agreement of the simulation and measurement data.

In this study, despite the consideration of various tooth depths for different electron energies, the dental configuration was the same to the previous study. Since build-up depth is not the same for different electron energies which were simulated in this study $(8,12$ and $14 \mathrm{MeV})$, therefore the depth of the tooth was selected based on the electron energy. In other words, the tooth was defined to be after the build-up depth in each energy. Based on this selection, the depth of the tooth was assumed to be $3.2 \mathrm{~cm}$ in $8 \mathrm{MeV}, 4.5 \mathrm{~cm}$ in $12 \mathrm{MeV}$ and $5.1 \mathrm{~cm}$ in $14 \mathrm{MeV}$ electron energies. In this study, the validated simulations were used in order to assess the impact of the tooth and dental restoration materials causing neutron contamination.

\section{Tooth and Restoration Materials}

The geometry of phantom was assumed to be as a cylinder having the diameter of 10 $\mathrm{cm}$ and height of $10 \mathrm{~cm}$ cylinder, and it was composed of soft tissues based on the compositions report (report No. 44) by the International Commission on Radiation Units and Measurements (ICRU) [13]. The dental restoration materials were the same as a previous study [11]: Ceramco C3 veneer, Eclipse and amalgam. The composition of tooth was assumed as $20 \%$ enamel and $80 \%$ dentine. The depth of tooth was assumed to be $3.2 \mathrm{~cm}$ with $8 \mathrm{MeV}, 4.5 \mathrm{~cm}$ with $12 \mathrm{MeV}$ and $5.1 \mathrm{~cm}$ with $14 \mathrm{MeV}$ electron energies [14].

\section{Neutron Contamination Dose Calcu- lation}

Neutron contamination due to the tooth and the restored tooth with dental material was scored for the 8 and 12 and $14 \mathrm{MeV}$ electrons from a Siemens Primus linear accelerator using MCNPX (2.6.0) simulation code. To know more information about the details of simulation and the composition of different restoration materials used in this study, it's suggested that the previously published report be reviewed [14].

Tooth and dental restoration material were located in the path of the central axis of the beam and the dose was obtained in $3 \times 3 \times 1$ $\mathrm{cm}^{3}$ voxels at different depths before and after the tooth. The neutron fluence was calculated using F4 tally to obtain the neutron contamination dose in these voxels. Since direct scoring of neutron contamination dose was timeconsuming, to score the neutron dose, the neutron fluence was scored, and then it was multiplied by fluence to dose conversion factor. This factor depends on energy, therefore to have accurate calculations, neutron fluence was computed in different energy bins and the neutron contamination dose was scored through the multiplication of neutron fluence by the fluence to dose conversion factor in each energy bin. The conversion factors were adopted from the NCRP 38 report [15].

In the calculations, the only variance reduction method used was energy cut-off for photons and electrons. Photons and electrons with energies less than $7 \mathrm{MeV}$ were not tracked, but neutrons were tracked until their energy reached to zero. To reduce the variance, each simulation program was run for $2 \times 10^{9}$ neutron histories and then each program was run 30 times with different seed numbers. Totally, $6 \times 10^{10}$ neutron histories were tracked for each program combination of tooth and the dental 
restoration at various amounts of energy. The maximum Monte Carlo uncertainty for these calculations was $22.0 \%$.

The relative neutron dose was defined by the definition of the ratio of: neutron dose deposited in a dose calculation cell in phantom with the presence of tooth/restored tooth to neutron dose in the same cell without the tooth.

\section{Results}

The relative doses of neutron contamination in the case of tooth and three common dental materials in dentistry are shown in Figure 1. The dental materials include the tooth restored with Ceramco C3 veneer, tooth restored with Eclipse and tooth restored with amalgam. Figure 1 is related to 8 and 12 , and $14 \mathrm{MeV}$ electron beams of Siemens Primus linear accelerator. This figure demonstrates changes in the relative neutron dose in various depths above and beyond the tooth. The relative neutron dose was calculated as the ratio of neutron dose in a calculation cell in the presence of a restoration material in the phantom to that in the absence of the restoration material.

The numerical values of the relative neutron contamination dose for the tooth and the mentioned restoration materials in different depths are presented in Table 1, those points which are positioned before the tooth are specified with star

\section{Discussion}

As a general result, the tooth or restored tooth simulated in this study changes the neutron contamination dose. Based on the results which are listed in Table 1, the relative neutron dose values are often more than unity, and this indicates an increase in the neutron dose contamination in the case of tooth and various dental materials in different energies. Although in $8 \mathrm{MeV}$ beam a decrease in relative neutron contamination is observed in some depths for the tooth, the tooth restored by Eclipse and Ceramco. The reason is that the threshold energy required for photonuclear reaction in the most nucleus is $10 \mathrm{MeV}$ or greater.

According to Figure 1, as the depth increases, the relative neutron dose in the $14 \mathrm{MeV}$ electron energy decreased after the tooth and increased before it. In the $12 \mathrm{MeV}$ electron energy, the tooth is in combination with Eclipse, and Ceramco that the trend is relatively same to the $14 \mathrm{MeV}$ electron energy for before and after the tooth. In $8 \mathrm{MeV}$ electron energy, the relative neutron dose with the case of tooth and various dental materials increases with increasing depth after the tooth. In general, at most points before and after the tooth the trend
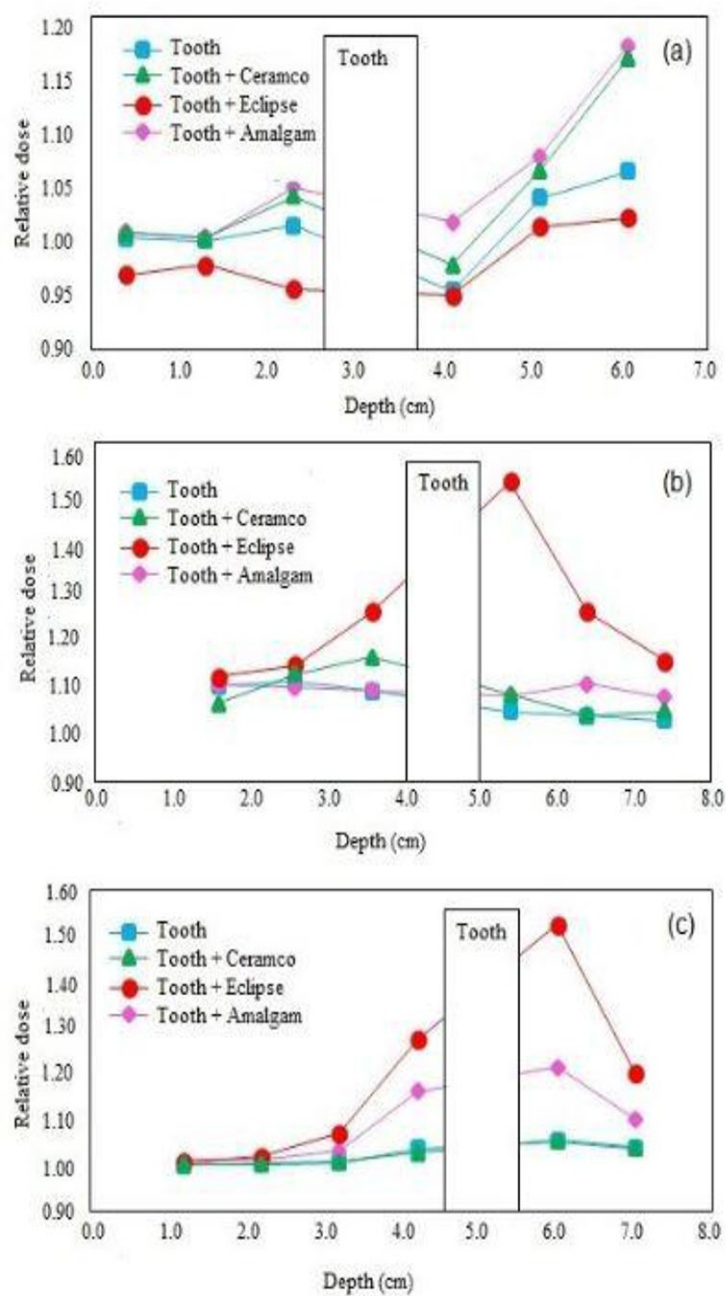

Figure 1: The relative neutron contamination dose in the case of tooth/restored tooth with Ceramco C3 veneer, Eclipse alloy and amalgam restoration materials. The data for the 8-14 MeV beams are shown in parts (a)(c), respectively 
Dental Restorations-neutron Contamination

Table 1: The relative neutron contamination dose in a phantom for the case of tooth/dental materials for 8-14 MeV beams.

$8 \mathrm{MeV}$

$12 \mathrm{MeV}$

$14 \mathrm{MeV}$

\begin{tabular}{|c|c|c|c|c|c|c|c|c|c|c|c|c|c|c|}
\hline  & 동 & 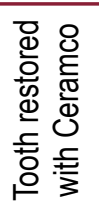 & 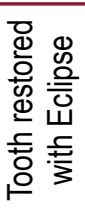 & 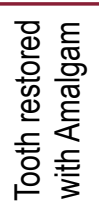 & $\frac{\widehat{E}}{\frac{\bar{E}}{0}}$ & 동 & 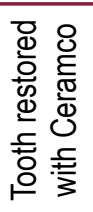 & 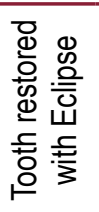 &  &  & 동 & 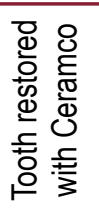 & 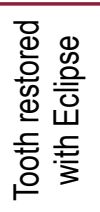 & 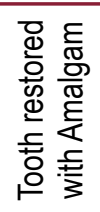 \\
\hline 0.4 & $1.00^{*}$ & $1.00^{*}$ & $0.97^{*}$ & $1.01^{*}$ & 1.6 & $1.10^{*}$ & $1.06^{*}$ & $1.12^{*}$ & $1.10^{*}$ & 1.2 & $1.00^{*}$ & $1.00^{*}$ & $1.00^{*}$ & $1.01^{*}$ \\
\hline 1.3 & $1.00^{*}$ & $1.00^{*}$ & $0.98^{*}$ & $1.00^{*}$ & 2.6 & $1.11^{*}$ & $1.12^{*}$ & $1.14^{*}$ & $1.09^{*}$ & 2.2 & $1.00^{*}$ & $1.00^{*}$ & $1.02^{*}$ & $1.01^{*}$ \\
\hline 2.3 & $1.01^{*}$ & $1.04^{*}$ & $0.95^{\star}$ & $1.05^{*}$ & 3.6 & $1.09^{*}$ & $1.16^{*}$ & $1.25^{*}$ & $1.09^{*}$ & 3.2 & $1.01^{*}$ & $1.01^{*}$ & $1.07^{*}$ & $1.03^{*}$ \\
\hline 4.1 & 0.95 & 0.98 & 0.95 & 1.01 & 5.4 & 1.04 & 1.08 & 1.52 & 1.08 & 4.2 & $1.04^{*}$ & $1.03^{*}$ & $1.27^{*}$ & $1.16^{*}$ \\
\hline 5.1 & 1.04 & 1.06 & 1.01 & 1.07 & 6.4 & 1.04 & 1.04 & 1.25 & 1.10 & 6 & 1.05 & 1.05 & 1.53 & 1.21 \\
\hline 6.1 & 1.06 & 1.16 & 1.02 & 1.17 & 7.4 & 1.03 & 1.04 & 1.15 & 1.07 & 7 & 1.04 & 1.04 & 1.20 & 1.10 \\
\hline
\end{tabular}

for relative neutron contamination dose is increasing and only at some points, in the 12, $14 \mathrm{MeV}$ electron energies, after the tooth the trend is decreasing.

The highest neutron dose contamination was achieved for the combination of tooth and Eclipse in the $12,14 \mathrm{MeV}$ electron energies, and it can be due to having a high atomic number element in this material (gold with atomic number 79 is the dominant element in the composition of this combination compared to oxygen with atomic number of 8 in the composition of Ceramco and silver with atomic number of 47 in the composition of amalgam) or a higher mass density $\left(13.8 \mathrm{~g} / \mathrm{cm}^{3}\right.$ for this material compared to $2.6 \mathrm{~g} / \mathrm{cm}^{3}$ for Ceramco and $8 \mathrm{~g} / \mathrm{cm}^{3}$ for amalgam). Therefore, this combination will produce more neutron contamination than the other combinations of the tooth and dental materials.

The results obtained in the present study show that neutron contamination is less in a lower energy $(8 \mathrm{MeV})$, thus using the lower energy electron beam is recommended as much as possible to prevent the production of high neutron contamination in head and neck cancer treatment of patients with dental restoration. Among the three energies studied in this study, $8 \mathrm{MeV}$ electron energy is preferable in the case of having the same choice of energy.

Treatment of head and neck cancers may include cancer of nasopharynx, tongue or oral cavity. The results of this study indicate that the presence of dental material can increase the neutron contamination at different depths before and after the tooth in electron beams of a linear accelerator. To reduce this neutron contamination in soft tissue, the use of bolus is recommended between the tooth and soft tissue. Of course, the presence of this bolus changes the electron dose distribution, and this effect should be considered in the process of treatment planning for patients with a type of head and neck cancer. The thickness for the needed appropriate bolus varies in different energies, and according to the results, the thickness is required to completely absorb the neutron contamination increases with increasing energy. However, it is necessary to determine the depths before and after the tooth that the neutron contamination is presented to be able to determine the amount of thickness needed from the bolus. For example, based on the results of relative neutron contamination dose in the $14 \mathrm{MeV}$ electron energy (Table 1), $3 \mathrm{~cm}$ of bolus may be required to absorb neutron contamination in the soft tissue in this energy.

Based on a literature review, we could not 
find a similar study that evaluated the neutron contamination dose for the electron mode of a linear accelerator in the case of the tooth and restoration materials, hence it be possible to compare the current results.

\section{Conclusion}

The presence of tooth/dental materials in the path of electron beam of a linear accelerator will increase the neutron contamination dose. This increase in neutron dose is dependent on depth, kind of restoration material, as well as the energy of electron beam. Therefore, the production of neutron doses should be accounted in the process of treatment planning while the tooth is irradiated with an electron beam. To reduce neutron dose to soft tissues before tooth, using a lower energy electron beam and putting up bolus between soft tissue and tooth will be recommended if they are possible. Furthermore, treatment planning should be planned by considering that the tooth with dental restoration materials should not be located in the beam path for the electron beam.

\section{Acknowledgment}

The authors would like to extend their highest gratitude to Shahid Beheshti University of Medical Sciences (SBMU) for the funds required for the current project.

\section{Conflict of Interest}

\section{None}

\section{References}

1. Heron M, Anderson RN. Changes in the Leading Cause of Death: Recent Patterns in Heart Disease and Cancer Mortality. NCHS Data Brief. 2016:1-8. PubMed PMID: 27598767.

2. Siegel RL, Miller KD, Jemal A. Cancer Statistics, 2017. CA Cancer J Clin. 2017;67:7-30. doi: 10.3322/caac.21387. PubMed PMID: 28055103.

3. In: National Cancer Institute at the National Institutes of Health. [2017]. Available from: https:// www.cancer.gov/about-cancer/treatment/types.

4. Barnett GC, West CM, Dunning AM, Elliott RM, Coles CE, Pharoah PD, et al. Normal tissue reactions to radiotherapy: towards tailoring treatment dose by genotype. Nat Rev Cancer. 2009;9:134-42. doi: 10.1038/nrc2587. PubMed PMID: 19148183. PubMed PMCID: PMC2670578.

5. Ding M, Newman F, Raben D. New radiation therapy techniques for the treatment of head and neck cancer. Otolaryngol Clin North Am. 2005;38:37195. doi: 10.1016/j.otc.2004.10.009. PubMed PMID: 15823599.

6. Kahn F, Gibbons J. Khan's the physics of radiation therapy. Philadelphia, PA: Lippincott Williams and Wilkins; 2014:39-57.

7. McKeown J, Schneider H, Schriber S, editors. High power operation of two side-coupled standing wave linac structures. Proceedings of the Proton Linear Accelerator Conference; Los Alamos; 1972.

8. Fraser J, Kidner S, McKeown J, McMichael G, editors. The Chalk River electron test accelerator. Proceedings of the Proton Linear Accelerator Conference; Los Alamos; 1972.

9. Brenner M, editor. Neutron Contamination of Electron Beams. Symposium on High-Energy Electrons; Berlin, Heidelberg: Springer; 1965.

10. Abdul Aziz M, Yusoff A, Salikin M. Monte Carlo electron beam dose distribution near high density inhomogeneities interfaces. World Acad Sci Eng Technol. 2011;58:338-41.

11. Bahreyni Toossi MT, Ghorbani M, Akbari F, Mehrpouyan M, Sobhkhiz Sabet L. Evaluation of the effect of tooth and dental restoration material on electron dose distribution and production of photon contamination in electron beam radiotherapy. Australas Phys Eng Sci Med. 2016;39:113-22. doi: 10.1007/s13246-015-0404-z. PubMed PMID: 26581762.

12. Bahreyni Toossi MT, Ghorbani M, Akbari F, et al. Monte Carlo simulation of electron modes of a Siemens Primus linac (8, 12 and $14 \mathrm{MeV})$. Journal of Radiotherapy in Practice. 2013;12:352-9. doi. 10.1017/S1460396912000593.

13. International Commission on Radiation Units and Measurements. Tissue substitutes in radiation dosimetry and measurement. ICRU Report 44: Bethesda; 1989.

14. Bahreyni Toossi MT, Behmadi M, Ghorbani M, Gholamhosseinian H. A Monte Carlo study on electron and neutron contamination caused by the presence of hip prosthesis in photon mode of a 15 MV Siemens PRIMUS linac. J Appl Clin Med Phys. 2013;14:52-67. doi: 10.1120/jacmp. v14i5.4253. PubMed PMID: 24036859. PubMed PMCID: PMC5714559.

15. National Council on Radiation Protection and Measurements [Internet]. Report 38, Protection Against Neutron Radiation (1971). Available from: http://www.ncrppublications.org/Reports/038. 